\title{
On some non-conformal fractals
}

\author{
Michał Rams* \\ Institute of Mathematics, Polish Academy of Sciences \\ ul. Śniadeckich 8, 00-950 Warszawa, Poland \\ e-mail: rams@impan.gov.pl
}

\begin{abstract}
This paper presents a simple method of calculating the Hausdorff dimension for a class of non-conformal fractals.
\end{abstract}

\section{Introduction}

An iterated function scheme acting on a complete metric space $X$ is a finite family of contracting maps $\mathcal{F}=\left\{f_{k}\right\}_{k=1}^{n} ; f_{k}: X \rightarrow X$. As noted by Hutchinson $[\mathrm{Hu}]$, the related multimap

$$
F(\cdot)=\bigcup_{k=1}^{n} f_{k}(\cdot)
$$

(acting on the space $B(X)$ of nonempty compact subsets of $X$, considered with the Hausdorff metric) is also a contraction. Hutchinson proved that if $X$ is complete, so is $B(X)$. Hence, by the Banach fixed point theorem, there exists a unique nonempty compact set $\Lambda$ satisfying

$$
\Lambda=F(\Lambda)=\lim _{n \rightarrow \infty} F^{n}(A) .
$$

The limit does not depend on the choice of $A \in B(X)$. $\Lambda$ is called the limit set of the iterated function scheme $\mathcal{F}$.

*supported by EU FP6 Marie Curie programme SPADE 2 and by Polish MNiSW Grant NN201 022233 'Chaos, fraktale i dynamika konforemna'

1991 Mathematics Subject Classification: 28A78, 28A80 
By similar reasoning, if we have a finite number of iterated function schemes $\left\{\mathcal{F}_{i}\right\}_{i=1}^{m}$ acting on $X$ and apply them in any order, the pointwise limit

$$
\Lambda_{\omega}=\lim _{n \rightarrow \infty} F_{\omega_{1}} \circ \ldots \circ F_{\omega_{n}}(A)
$$

exists for all $\omega \in \Omega=\{1, \ldots, m\}^{\mathbb{N}}$ and does not depend on $A \in B(X)$.

The question we want to answer (motivated by [Lu], see also [N], GL], GL2] and the incoming paper [Re] is: when the iterated function schemes $\mathcal{F}_{i}$ are of some special class (for which we can calculate the Hausdorff dimension of the limit set of any deterministic iterated function scheme from this class) and the sequence $\omega$ is chosen, what will be the value of the Hausdorff dimension of $\Lambda_{\omega}$ ?

We will present a simple method of dealing with this question, working for Lalley-Gatzouras maps [LG], Barański maps [B] and higher dimensional affine-invariant sets of Kenyon and Peres [KP]. The only assumption about $\omega$ we need is that each symbol $i$ has a limit frequency of appearance. For simplicity, we will only present the proof for an example: a class of iterated function schemes considered by Lalley and Gatzouras.

We refer the reader interested in other non-conformal random iterated constructions to [F], GL2] and references therein.

\section{Lalley-Gatzouras schemes}

The Lalley-Gatzouras scheme $\mathcal{F}$ is a self-affine IFS given by a family of maps

$$
f_{i, j}(x, y)=\left(a_{i j} x+c_{i j}, b_{i} y+d_{i}\right),
$$

where the alphabet $A$ of allowed symbols is $1 \leq i \leq m_{1}, 1 \leq j \leq m_{2}(i)$. We will assume that $b_{i} \geq a_{i j}$ (that is, the contraction in the horizontal direction is not weaker than the contraction in the vertical direction for all maps).

We will also assume that for all $(i, j) \in A 0<a_{i j}<1,0 \leq c_{i 1}<\ldots<$ $c_{i m_{2}(i)} \leq 1-a_{i m_{2}(i)}, c_{i j+1} \geq a_{i j}+c_{i j}$ and that $0<b_{i}<1,0 \leq d_{1}<\ldots<$ $d_{m_{1}} \leq 1-b_{m_{1}}, d_{i+1} \geq b_{i}+d_{i}$. We will say that the separation condition holds if we actually have $c_{i j+1}>a_{i j}+c_{i j}$ and $d_{i+1}>b_{i}+d_{i}$.

The main result of [LG] is the formula for the Hausdorff dimension of the limit set $\Lambda$ :

$\operatorname{dim}_{H}(\Lambda)=\max \left\{\frac{\sum_{i} \sum_{j} p_{i j} \log p_{i j}}{\sum_{i} \sum_{j} p_{i j} \log a_{i j}}+\sum_{i} q_{i} \log q_{i}\left(\frac{1}{\sum_{i} q_{i} \log b_{i}}-\frac{1}{\sum_{i} \sum_{j} p_{i j} \log a_{i j}}\right)\right\}$, 
where $\left\{p_{i j}\right\}$ is a probability distribution on $A, q_{i}=\sum_{j} p_{i j}$ and the maximum is over all possible $\left\{p_{i j}\right\}$.

Consider now a family of Lalley-Gatzouras schemes $\left\{\mathcal{F}_{k}\right\}_{k=1}^{m}$ with alphabets $A_{k}$ and maps $f_{i, j}^{(k)}$. As mentioned above, we can apply them in any order $F_{\omega_{1}} \circ F_{\omega_{2}} \circ \ldots, \omega=\omega_{1} \omega_{2} \ldots \in \Omega=\{1, \ldots, m\}^{\mathbb{N}}$ and obtain some limit set $\Lambda_{\omega}$. We will assume that the limits

$$
P_{k}=\lim _{n \rightarrow \infty} \frac{1}{n} \sharp\left\{1 \leq l \leq n ; \omega_{l}=k\right\}
$$

exist and are positive. We will ask what is the value of $\operatorname{dim}_{H}\left(\Lambda_{\omega}\right)$.

Before formulating the answer, let us note that any finite product $F_{\omega_{1}} \circ$ $\ldots \circ F_{\omega_{n}}$ is again a Lalley-Gatzouras scheme. It follows that we can calculate the Hausdorff dimension of $\Lambda_{\omega}$ for any periodic sequence $\omega$. Given a rational probabilistic vector $Q=\left(Q_{1}, \ldots, Q_{m}\right)$, we can choose a periodic sequence $\omega(Q)$ in which the frequency of symbol $k$ is $Q_{k}$. Let us write

$$
\mathrm{E}(Q)=\operatorname{dim}_{H} \Lambda_{\omega(Q)} .
$$

Our main result is as follows.

Theorem 2.1. The function $E(Q)$ is well defined, does not depend on the choice of $\omega(Q)$. We can extend it by continuity to the whole simplex of probabilistic vectors (we will keep the notation $E(Q)$ for the extended function). We have

$$
\operatorname{dim}_{H}\left(\Lambda_{\omega}\right)=E(P)
$$

\section{Proof of Theorem 2.1}

Let us start by presenting a more detailed description of $\Lambda_{\omega}($ compare $[\mathrm{Hu}]$ ). Let $A_{\omega}=A_{\omega_{1}} \times A_{\omega_{2}} \times \ldots$. We define a projection $\pi_{\omega}: A_{\omega} \rightarrow \Lambda_{\omega}$ by the formula

$$
\pi_{\omega}\left(\left(i_{1}, j_{1}\right),\left(i_{2}, j_{2}\right), \ldots\right)=\lim _{n \rightarrow \infty} f_{i_{1}, j_{1}}^{\left(\omega_{1}\right)} \circ \ldots \circ f_{i_{n}, j_{n}}^{\left(\omega_{n}\right)}(0,0),
$$

$\left(i_{k}, j_{k}\right) \in A_{k}$. We get

$$
\Lambda_{\omega}=\pi_{\omega}\left(A_{\omega}\right)
$$

Because of the nonconformality of the system, the most natural class of subsets of $A_{\omega}$ to study are not cylinders but rectangles (in particular, 
approximate squares). The rectangle is defined as follows: given a sequence $(i, j) \in A_{\omega}$ and two natural numbers $n_{1} \leq n_{2}$ we define

$$
R_{n_{1}, n_{2}}(i, j)=\left\{\left(i^{\prime}, j^{\prime}\right) \in A_{\omega} ; i_{k}^{\prime}=i_{k} \forall k \leq n_{2}, j_{k}^{\prime}=j_{k} \forall k \leq n_{1}\right\} .
$$

We will call

$$
d_{1}\left(R_{n_{1}, n_{2}}(i, j)\right)=\prod_{k=1}^{n_{1}} a_{i_{k} j_{k}}^{\left(\omega_{k}\right)}
$$

the width and

$$
d_{2}\left(R_{n_{1}, n_{2}}(i, j)\right)=\prod_{k=1}^{n_{2}} b_{i_{k}}^{\left(\omega_{k}\right)}
$$

the height of the rectangle $R_{n_{1}, n_{2}}(i, j)$. Indeed, the projection of a rectangle under $\pi_{\omega}$ is the intersection of $\Lambda_{\omega}$ with a geometric rectangle of the same width and of the same height. The rectangle of approximately (up to a constant) equal width and height is called an approximate square.

Our main step is the following proposition.

Proposition 3.1. For $Q$ a rational probabilistic vector close to $P$ and for any choice of $\omega(Q)$, we can construct a bijection $\tau: A_{\omega(Q)} \rightarrow A_{\omega}$ with the following properties. Let $R=R_{n_{1}, n_{2}}^{(\omega(Q))}(i, j)$ be an approximate square in $A_{\omega(Q)}$ of width $d$. If $\delta=\max \left|P_{k}-Q_{k}\right|$ is sufficiently small, $\tau(R)$ contains an approximate square of width at least $d^{1+K \delta+\varepsilon}$ and is contained in an approximate square of width at most $d^{1-K \delta-\varepsilon}, K$ depending only on the iterated schemes but not on $P$ or $Q$ and $\varepsilon$ arbitrarily small for sufficiently small $d$.

Proof. We will need the following simple statement (a reformulation of (2.1)):

Lemma 3.2. For every $n$ there exists $\varepsilon(n)$ such that for each $k$ the $n$ th appearance of symbol $k$ in the sequence $\omega$ takes place between positions $n / P_{k}\left(1-\varepsilon\left(n / P_{k}\right)\right)$ and $n / P_{k}\left(1+\varepsilon\left(n / P_{k}\right)\right)$. Moreover, $\varepsilon(n)$ goes monotonically to 0 as $n$ goes to $\infty$.

Consider now the pair of sequences: $\omega$, the sequence we work with, and $\omega(Q)$, a periodic sequence with frequencies $Q$. We will assume that $Q$ is $\delta$-close to $P$ and that both probabilistic vectors are positive. Obviously, in the sequence $\omega(Q)$ the $n$-th appearance of symbol $k$ is at position $n / Q_{k}$, give or take a constant.

We will define $\chi_{\omega, \omega(Q)}$ as a permutation of $\mathbb{N}$ in the following way: if $l_{1}$ is the place of $n$-th appearance of symbol $k$ in the sequence $\omega$ and $l_{2}$ is the place 
of $n$-th appearance of symbol $k$ in the sequence $\omega(Q)$, we set $\chi_{\omega, \omega(Q)}\left(l_{1}\right)=l_{2}$. We can then construct a bijection $\tau: A_{\omega(Q)} \rightarrow A_{\omega}$ as

$$
\tau\left(\left(i_{1}, j_{1}\right),\left(i_{2}, j_{2}\right), \ldots\right)=\left(i_{\chi_{\omega, \omega}(Q)}(1), j_{\chi_{\omega, \omega}(Q)}(1)\right) \ldots
$$

Denote

$$
D_{1}=\chi_{\omega, \omega(Q)}\left(\left\{1, \ldots, n_{1}\right\}\right)
$$

and

$$
D_{2}=\chi_{\omega, \omega(Q)}\left(\left\{n_{1}+1, \ldots, n_{2}\right\}\right)
$$

We remind that the rectangle $R$ is defined as the set of sequences $\left(i^{\prime}, j^{\prime}\right) \in$ $A_{\omega(Q)}$ for which we fix the first $n_{1}\left(i_{k}^{\prime}, j_{k}^{\prime}\right)$ and the following $n_{2}-n_{1} i_{k}^{\prime}$. Hence, the set $\tau(R)$ is the set of sequences $\left(i^{\prime}, j^{\prime}\right) \in A_{\omega}$ for which we fix $\left(i_{k}^{\prime}, j_{k}^{\prime}\right)$ for $k \in D_{1}$ and we fix $i_{k}^{\prime}$ for $k \in D_{2}$.

Denote

$$
\begin{gathered}
r_{1}=\inf \left(\mathbb{N} \backslash D_{1}\right)-1, \\
r_{2}=\inf \left(\mathbb{N} \backslash\left(D_{1} \cup D_{2}\right)\right)-1, \\
s_{1}=\sup \left(D_{1}\right), \\
s_{2}=\sup \left(D_{1} \cup D_{2}\right) .
\end{gathered}
$$

We have

$$
R_{s_{1}, s_{2}}^{(\omega)}(\tau(i, j)) \subset \tau(R) \subset R_{r_{1}, r_{2}}^{(\omega)}(\tau(i, j)) .
$$

Assume $\delta$ is much smaller than any $P_{k}$. By Lemma 3.2,

$$
\begin{aligned}
& r_{1} \geq n_{1}\left(1-K_{0} \varepsilon\left(n_{1}\right)-K_{0} \delta\right), \\
& r_{2} \geq n_{2}\left(1-K_{0} \varepsilon\left(n_{2}\right)-K_{0} \delta\right), \\
& s_{1} \leq n_{1}\left(1+K_{0} \varepsilon\left(n_{1}\right)+K_{0} \delta\right), \\
& s_{2} \leq n_{2}\left(1+K_{0} \varepsilon\left(n_{2}\right)+K_{0} \delta\right)
\end{aligned}
$$

for some $K_{0}>0$ depending only on the iterated schemes.

Consider the width of $R_{r_{1}, r_{2}}^{(\omega)}(\tau(i, j))$ versus the width of $R$. The latter is a product of $n_{1}$ numbers $a_{i j}^{(k)}$, the former it the subproduct of $r_{1}$ of those numbers. As all $a_{i j}^{(k)}$ are uniformly bounded away from 0 and 1 ,

$$
d_{1}\left(R_{r_{1}, r_{2}}^{(\omega)}(\tau(i, j))\right) \leq d^{1-K \varepsilon\left(n_{1}\right)-K \delta}
$$

for some uniformly chosen $K$, depending only on the iterated schemes. Similar reasoning proves 


$$
d_{2}\left(R_{r_{1}, r_{2}}^{(\omega)}(\tau(i, j))\right) \leq d^{1-K \varepsilon\left(n_{2}\right)-K \delta} .
$$

Consider now the width of $R_{s_{1}, s_{2}}^{(\omega)}(\tau(i, j))$ versus the width of $R$. The former a product of $s_{1}$ numbers $a_{i j}^{(k)}$, the latter it the subproduct of $n_{1}$ of those numbers, the same reasoning as before gives us

$$
\begin{aligned}
& d_{1}\left(R_{s_{1}, s_{2}}^{(\omega)}(\tau(i, j))\right) \geq d^{1+K \varepsilon\left(n_{1}\right)+K \delta}, \\
& d_{2}\left(R_{s_{1}, s_{2}}^{(\omega)}(\tau(i, j))\right) \geq d^{1+K \varepsilon\left(n_{2}\right)+K \delta} .
\end{aligned}
$$

The rectangles $R_{r_{1}, r_{2}}^{(\omega)}(\tau(i, j))$ and $R_{s_{1}, s_{2}}^{(\omega)}(\tau(i, j))$ are not necessarily approximate squares, but we can easily replace the former by some slightly larger rectangle which is an approximate square and we can replace the latter by some slightly smaller rectangle which is an approximate square. We are done.

Remark. We can introduce a metric on $A_{\omega}$, defining the distance between two points as the sum of width and height of the smallest rectangle containing them both. This metric is natural because if the maps satisfy separation condition, $\pi_{\omega}$ is bi-Lipschitz (without separation condition it will only be a Lipschitz projection). In this metric, the maps $\tau, \tau^{-1}$ are Hölder continuous with every exponent smaller than 1 (if $\delta=0$ ) or with exponent $1-K \delta$ (if $\delta$ is positive but small).

This proposition basically ends the proof of Theorem 2.1. By Proposition 3.3 and Lemma 5.2 in [LG], for any Lalley-Gatzouras scheme there exists a probabilistic measure $\mu$ supported on $A^{\mathbb{N}}$ such that

i) for a $\mu$-typical point $(i, j)$ and the decreasing sequence of all approximate squares $R_{k}=R_{n_{1}(k), n_{2}(k)}(i, j)$,

$$
\frac{\log \mu\left(R_{k}\right)}{\log d_{1}\left(R_{k}\right)} \rightarrow \operatorname{dim}(\Lambda)
$$

ii) for every point $x \in A^{\mathbb{N}}$ there exists a decreasing sequence of approximate squares $R_{k}=R_{n_{1}(k), n_{2}(k)}(i, j)$ for which

$$
\frac{\log \mu\left(R_{k}\right)}{\log d_{1}\left(R_{k}\right)} \rightarrow \operatorname{dim}(\Lambda)
$$

We can define such measure $\mu_{Q}$ supported on $A_{\omega(Q)}$ for any rational $Q$ (because this is again a Lalley-Gatzouras scheme). We can then transport this measure to $A_{\omega}$ by the map $\tau$. We obtain a measure $\nu_{Q}$ such that 
i) for a $\nu_{Q}$-typical point $(i, j)$ and the decreasing sequence of all approximate squares $R_{k}=R_{n_{1}(k), n_{2}(k)}(i, j)$,

$$
\liminf \frac{\log \nu_{Q}\left(R_{k}\right)}{\log d_{1}\left(R_{k}\right)} \geq \mathrm{E}(Q)(1-K \delta),
$$

ii) for every point $x \in A_{\omega}$ there exists a decreasing sequence of approximate squares $R_{k}=R_{n_{1}(k), n_{2}(k)}(i, j)$ for which

$$
\lim \sup \frac{\log \nu_{Q}\left(R_{k}\right)}{\log d_{1}\left(R_{k}\right)} \leq \mathrm{E}(Q)(1+K \delta) .
$$

It implies that

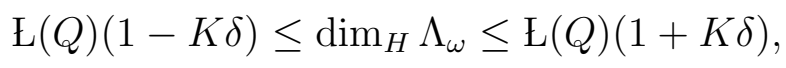

the proof is as in $[\mathrm{LG}]$.

This result has immediate applications for random systems, obtained by choosing $\omega$ randomly with respect to some Bernoulli measure on $\Omega$.

On the other hand, this method is not going to work for stochasticallyselfsimilar systems considered in $[\mathrm{F}]$ or $\mathrm{GaL}$. For such systems we would not have a single sequence $\omega$ but instead $\omega$ would depend on the point in the fractal. While we would still be able to define $\tau$ almost everywhere, the sequences $\omega(x)$ at different points $x \in \Lambda$ would not all satisfy Lemma 3.2, and hence $\tau$ would not everywhere have nice Hölder properties.

\section{References}

[B] K. Barański, Hausdorff dimension of the limit sets of some planar geometric constructions, Adv. Math. 210 (2007), 215-245.

[F] K. Falconer, Fractal geometry. Mathematical foundations and applications. John Wiley \& Sons, Hoboken 2003.

[GaL] D. Gatzouras, S. P. Lalley, Statistically self-affine sets: Hausdorff and box dimensions, J. Theor. Prob. 7 (1994), 437-468.

[GL] Y. Gui, W. Li, A random version of McMullen-Bedford general Sierpinski carpets and its application, Nonlinearity 21 (2008), 1745-1758.

[GL2] Y. Gui, W. Li, Multiscale self-affine Sierpinski carpets, Nonlinearity 23 (2010), 495-512. 
[Hu] J. E. Hutchinson, Fractals and self-similarity, Indiana Univ. Math. J. 30 (1981), 713-747.

[KP] R. Kenyon, Y. Peres, Measures of full dimension on affine-invariant sets. Erg. Th. Dyn. Sys. 16 (1996), 307-323.

[LG] S. P. Lalley, D. Gatzouras, Hausdorff and box dimensions of certain self-affine fractals, Indiana Univ. Math. J. 41 (1992), 533-568.

[Lu] N. Luzia, Hausdorff dimension of certain random self-affine fractals, preprint.

[N] O. A. Nielsen, The Hausdorff and Packing Dimensions of Some Sets Related to Sierpinski Carpets, Canad. J. Math. 51 (1999), 1073-1088.

[Re] H. Reeve, Multifractal analysis of Birkhoff averages on some nonconformal poly iterated function systems, in preparation. 